\title{
Statistical Based Vectorization for Standard Vector Graphics
}

\author{
Sebastiano Battiato, Giovanni Maria Farinella, and Giovanni Puglisi \\ Dipartimento di Matematica e Informatica, University of Catania, Italy \\ Image Processing Laboratory \\ \{battiato, gfarinella, puglisi\}@dmi.unict.it \\ http://www.dmi.unict.it/ iplab
}

\begin{abstract}
In this paper a novel algorithm for raster to vector conversion is presented. The technique is mainly devoted to vectorize digital picture maintaining an high degree of photorealistic appearance specifically addressed to the human visual system. The algorithm makes use of an advanced segmentation strategy based on statistical region analysis together with a few ad-hoc heuristics devoted to track boundaries of segmented regions. The final output is rendered by Standard Vector Graphics. Experimental results confirm the effectiveness of the proposed approach both in terms of perceived and measured quality. Moreover, the final overall size of the vectorized images outperforms existing methods.
\end{abstract}

\section{Introduction}

The vectorial format is useful for describing complex graphical scenes using a collection of graphic vectorial primitives, offering the typical advantages of vectorial world: scalability, resolution independence, etc. In this paper we propose a novel technique to cover the gap between the graphical vectorial world and the raster real world typical of digital photography. Standard Vector Graphics (SVG) is a standard language for describing two-dimensional graphics in XML ([1]). This format could be very useful in the world of mobile imaging device where the typical capability of a camera needs to match with limited colour/dimension resolutions display. Another potential application is presented in 2] where an ad-hoc method for icon vectorization is applied emphasizing the good performances in terms of resolution scalability.

Recently, some commercial and free software have been developed using some solution to the "raster to SVG" problem (Vector Eye [3], Autotrace 4], Kvec [5]). Almost all of them are devoted to SVG rendering of graphic images (e.g. clip art, etc.) showing in such case good performances but also several perceptual drawbacks when applied to digital pictures.

Considering photorealistic vectorization, a few advanced approaches are described in SWaterG [6], SVGenie and SVGWave [7, where ad-hoc segmentation strategies, making use respectively of watershed decomposition and adaptive triangulation, allow to obtain good performances both in terms of perceptual accuracy and overall compression size. An useful review is presented in [8]. We 
also mention the work presented in [9] where a region-contour segmentation scheme that takes into account contours resulting from colour gradients in an image, as well as their interrelationships in terms of bounding a common region, is described. One of the main drawback of almost all cited approaches is the parameters tuning with respect to the final application. The correct trade-off between final perceived quality, scalability and the corresponding file size is a very challenging task, mainly for user that doesn't know the algorithmic details of each solution. Our proposed technique is based on a single input parameter, that fixes the degree of "coarseness" to be used in the segmentation step. An efficient algorithm has been used for the preliminary analysis of input raster data: Statistical Region Merging (SRM) [10. Such segmentation algorithm is able to capture the main structural components of a digital image using a simple but effective statistical analysis.

Our technique consists of two main steps: the image is partitioned in polygonal regions using SRM; the borders of segmented regions are properly tracked and described by SVG primitives making also use of some smart tips.

The final results show how the proposed strategy outperforms state-of-art techniques in terms of visual perception, measured image quality and compression size. The overall performances are described in terms of Rate-Distortion plots, by varying the unique involved quality parameter.

The rest of the paper is organized as follows. In Section 2 we briefly review the main details of SRM. The successive Section describes how boundary representation is computed while Section 4 reports the generation of SVG representation by using suitable mapping strategies. In Section 5 several experimental results together with some comparisons with other techniques are reported. A final Section closes the paper tracking also direction for future works.

\section{$2 \quad$ Statistical Region Merging}

Segmentation is the process of partitioning an image into disjoint and homogeneous regions. A more formal definition can be given in the following way [1]: let $I$ denote an image and let $H$ define a certain homogeneity predicate; the segmentation of $I$ is a partition $P$ of $I$ into a set of $N$ regions $R_{1}, R_{2}, \ldots, R_{N}$, such that: $\bigcup_{n=1}^{N} R_{n}=I$ with $R_{n} \cap R_{m}=\emptyset, n \neq m ; H\left(R_{n}\right)=$ true $\forall n$; $H\left(R_{n} \cup R_{m}\right)=$ false $\forall R_{n}$ and $R_{m}$ adjacent.

Recently, thanks to the increasing speed and decreasing cost of computation, many advanced techniques have been developed for segmentation of colour images. In particular we used the Statistical Region Merging [10] algorithm that belongs to the family of region growing techniques with statistical test for region fusion. SRM is based on the follow model of image: $I$ is an image with $|I|$ pixels each containing three values $(\mathrm{R}, \mathrm{G}, \mathrm{B})$ belonging to the set $\{1,2, \ldots, \mathrm{g}\}$. The model considers image $I$ as an observation of perfect unknown scene $I^{*}$ in which pixels are represented by a family of distributions from which each colour level is sampled. In particular, every colour level of each pixel of $I^{*}$ is described by a set of $\mathrm{Q}$ independent random variables with values in $[0, \mathrm{~g} / \mathrm{Q}]$. In $I^{*}$ the optimal 
regions satisfy the following homogeneity properties: inside any statistical region and for any colour channel, statistical pixels have the same expectation value for this colour channel; the expectation value of adjacent regions is different for at least one colour channel. From this model a suitable merging predicate is applied as described in [10].

The order in which the tests of merging were done follows a simple invariant $A$ : when any test between two true regions occurs, that means that all tests inside each region have previously occurred.

In the experiments, $A$ is approximated by a simple algorithm based on gradient of nearby pixels. In particular Nielsen and Nock consider a function $f$ defined as follow:

$$
f\left(p, p^{\prime}\right)=\max _{a \in R, G, B} f_{a}\left(p, p^{\prime}\right) .
$$

A simple choice for $f_{a}$ is:

$$
f_{a}\left(p, p^{\prime}\right)=\left|p_{a}-p_{a}^{\prime}\right|
$$

More complex function that extends classical edge detection convolution kernels could be used to define $f_{a}$. In our case we used the Sobel gradient mask.

The pseudo-code of algorithm is the following:

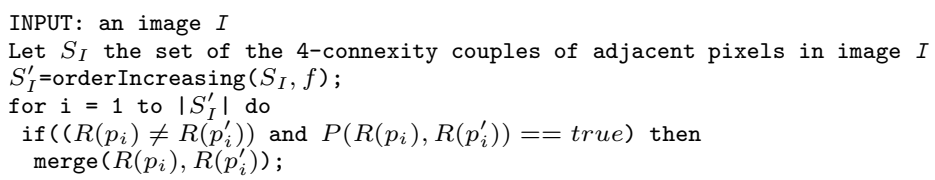

The set of the pairs of adjacent pixel $\left(S_{I}\right)$ is sorted according to the value of (1). Afterwards the algorithm takes every couple of pixels $\left(p, p^{\prime}\right)$ of $S_{I}$ and if the regions to which they belong $\left(R(p)\right.$ and $\left.R\left(p^{\prime}\right)\right)$ were not the same and satisfactory the merging predicate, it merges the two regions. SRM algorithm gives, for each segmented region, the list of pixel belonging to it and the related mean colour. We use this output as starting point to create a vectorial representation of image.

\section{Contouring}

To obtain a vectorial representation we have to find the border of segmented regions. This could be done more easily if we consider the pixels belonging to several groups (Fig. 1(a)p. First of all pixels are divided in:

- internal pixels: pixels with all neighbours (in a 4-connexity scheme) belonging to the same region;

- border pixels: remaining pixels.

Due to the overall complexity of border regions a further setting into two groups is required:

- close pixels: pixels with at least an internal pixel as neighbour (in a 8connexity scheme);

- open pixels: remaining pixels. 
After having assigned each pixel to the corresponding category we describe regions in vectorial form. In particular there are two types of curves: close curves and open curves. In both cases we could approximate their boundaries through segments with eight possible directions (Fig. $1(\mathrm{~d})$.

A close curve is a curve made up of only close pixels. Initially we consider a simple configuration (Fig. 1(b) to explain how segments could be found from close pixels list. The pseudo-code that describes the algorithm is the following:

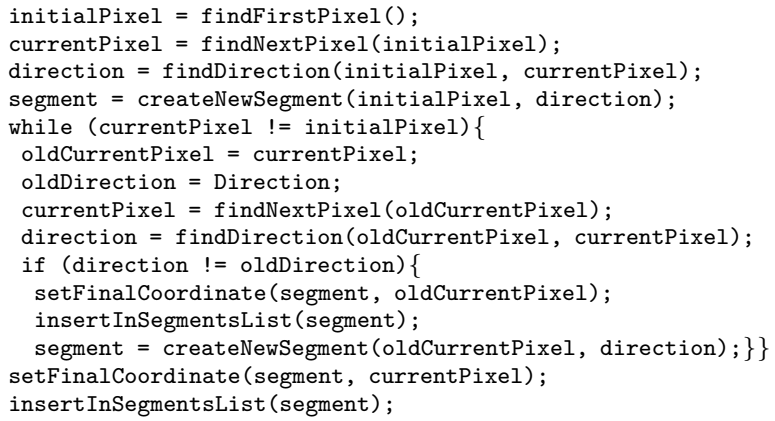

The functions are:

- findFirstPixel(): it chooses the top-left pixel as initial pixel.

- findNextPixel (currentPixel): it looks for the next pixel in the neighbourhood following a counter clockwise direction.

- createNewSegment(oldCurrentPixel, direction): it creates a new segment with first coordinate oldCurrentPixel and direction direction.

- setFinalCoordinate (segment, oldCurrentPixel): it sets the final coordinate of segment segment at oldCurrentPixel.

- insertInSegmentList (segment): it adds segment in the list of segments that describes the close curve.

Our algorithm chooses the top-left pixel of curve as initial pixel and following the boundary in counter clockwise direction creates the segments necessary for a vectorial description. There are a series of complex configuration: regions with internal curve; ambiguity in the choice of next currentPixel; several close curve belonging to the same region.

To properly consider these cases it is needed to slightly modify the algorithm described above. The full description and details are reported at http://www.dmi.unict.it/ iplab.

\subsection{Open Curve}

Even if a good segmentation algorithm should create regions with simple boundaries and not ragged this is not always the case. For this reason we have divided border pixels into two groups: close pixels and open pixels. The last are the pixels devoted to describe the ragged above mentioned. For simple configurations (Fig. 1(c) we could use the following algorithm: 


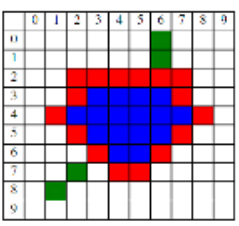

(a)

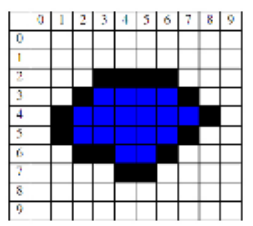

(b)

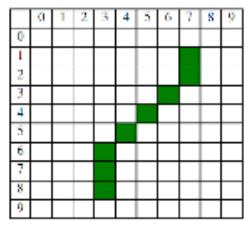

(c)

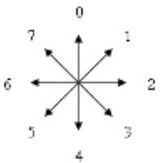

(d)

Fig. 1. An example of different kind of pixels: internal (blue), close (red), and open (green) (a). Example of simple close (b) and open curve (c). Possible directions of segments that approximate the boundaries of regions $(d)$.

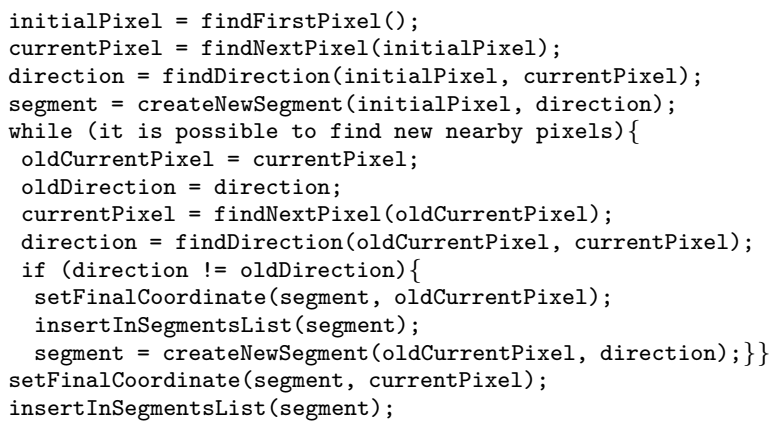

It is very similar to the one described for the close curve, but the following function has a different behaviour:

- findFirstPixel(): it choices a pixel with only a neighbour as initial pixel. Moreover when a pixel is chosen it is deleted from the list of open pixels.

For complex configuration we used a more sophisticated algorithm. Full description with more details are available at the following web address http:// www.dmi . unict.it/ iplab.

\section{SVG Generation Code}

After tracking the curve boundaries is necessary to map the data by SVG primitives. In particular a generic close curve could be represented in the following way:

<path d="M x1,x1 L x2,y2 Lx3,y3 Z" stroke ="\#RRGGBB" fill"\#RRGGBB" />

where $\mathrm{x} 1, \mathrm{y} 1, \mathrm{x} 2, \mathrm{y} 2, \mathrm{x} 3, \mathrm{y} 3$ are the vertexes coordinates and $\mathrm{RR}, \mathrm{GG}, \mathrm{BB}$ are respectively the hex representation of red, green, blue mean value of the region that close curve belong to. An open curve could be represented in the following way: 
Open curves are no filled ( $\mathrm{fill}=$ "none") and start point is not equal to final point ( $\mathrm{Z}$ parameter is absent). In order to obtain small file size some optimization could be done [12]:

- path element permits to eliminate some separator characters, to use relative coordinate (m, I command) and $\mathrm{h}, \mathrm{v}$ command for horizontal and vertical lines respectively;

$-\langle g\rangle$ elements is used to properly ensemble common graphic properties of various primitives.

\section{$5 \quad$ Experimental Results}

We have done several experiments in order to study the behaviour of our solution as the Q parameter increases. In particular we used two indexes of performance: PSNR and bit per pixel. The first index is tied with the quality of the image, the second with the file size.

We use "Lena" image to show the relation between Q parameter and some indexes of performance (PSNR, bpp). Analogous results have been found for other images. Figures $(2(\mathrm{a})$ and $(2(\mathrm{~b})$ show that both quality and image size grow as the $\mathrm{Q}$ parameter increases. In fact increasing $\mathrm{Q}$, SRM produces more regions and more details need to be represented. Moreover it is not useful increasing the Q parameter over a certain threshold (see Fig. 2(c)].

For sake of comparison SVG images obtained with our algorithm have been compared with other techniques such as Vector Eye [3], SWaterG [6], SVGenie and SVGWave 7]. The set of images used for comparison are of different kinds: graphics, textual, colours and grey levels. The parameters of different techniques have been set in order to obtain images visually agreeable. Figure $3(\mathrm{a})$ shows

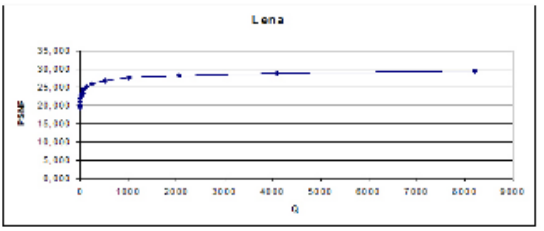

(a) Relation between PSNR and Q.

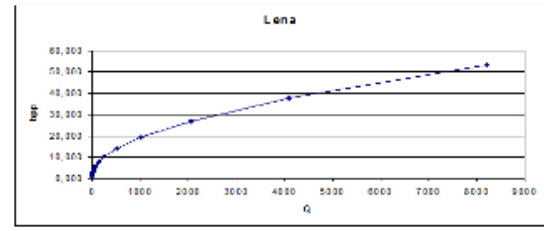

(b) Relation between bpp and Q.

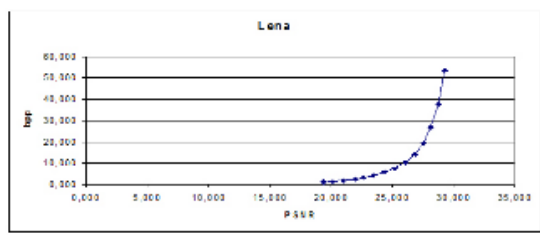

(c) Relation between PSNR and bpp.

Fig. 2. Relation between Q parameter and some indexes of performance (PSNR, bpp) for Lena image 


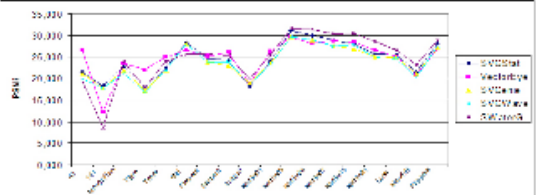

(a) PSNR comparison of dataset images outputs.

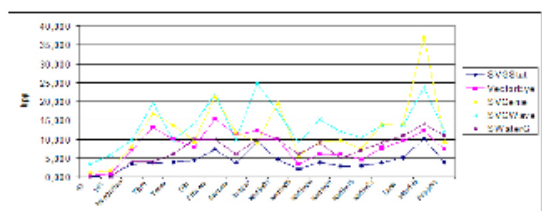

(c) Bit per pixels comparison of dataset images compressed outputs (SVGZ embedded coding).

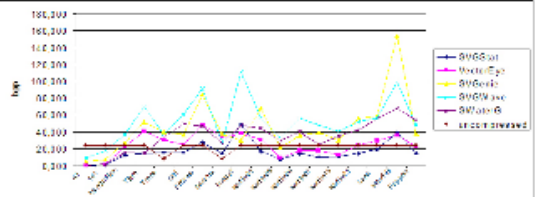

(b) Bit per pixels comparison of dataset images outputs.

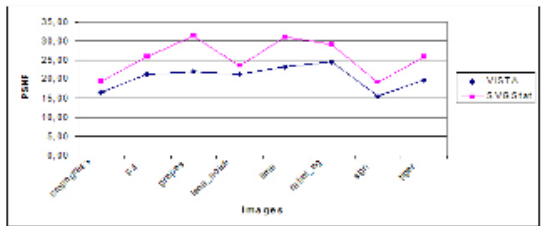

(d) PSNR comparison of our solution with respect to VISTA dataset images outputs.

Fig. 3. Comparison between different techniques
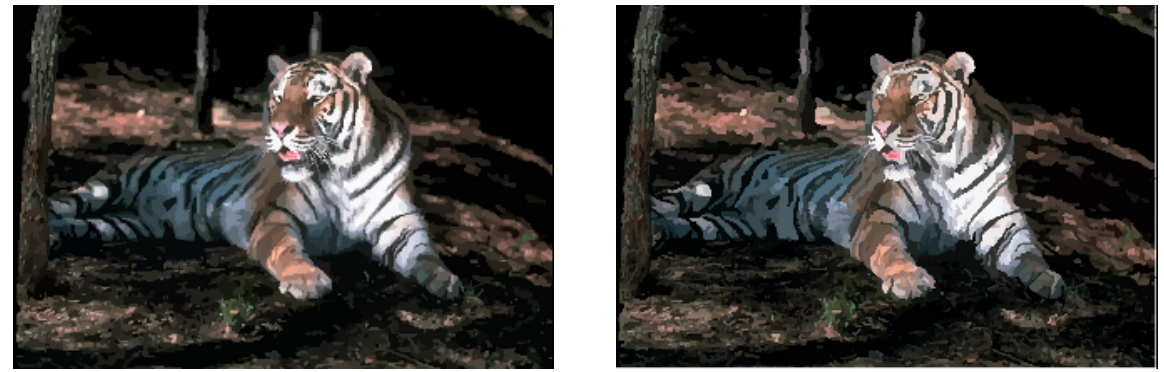

Fig. 4. Visual comparison between tiger image vectorized with our solution (left) (99 $\mathrm{KB}, \mathrm{PSNR}=26,51$ ) and VISTA SVG image (right) (94 KB, PSNR=19,69). The file size is almost the same but the quality of image produced with our technique is clearly better.

similar PSNR values for all the techniques. Fixing the quality, the file size produced by different techniques changes. In particular our algorithm outperforms the others (see Fig. 3(b) and 3(c) producing files of lower size.

A different kind of experiment has been done with the recent VISTA technique 9. In particular we have used for suitable comparison the data (original, SVG code) downloadable from http://www.svgopen.org/2005/papers/Prasad_Abstract_R2V4SVG. For sake of comparison we fix the overall bit size of each image in order to properly tune our Q parameter. The final quality, measured (PSNR) and perceptual, is clearly better then corresponding VISTA images as showed in Fig. (3(d) and (4). It is useful to consider that our algorithm has only a parameter $(\mathrm{Q})$ that is easily tuneable to obtain the desired quality-size trade-off. Further experiments can be found at the following web address: http://www.dmi.unict.it/ iplab. 


\section{Conclusion and Future Works}

In this paper we have proposed a novel technique able to convert raster images in a SVG format. Moreover, we have carried out several experiments showing that our algorithm outperforms other similar techniques. Future researches will be devoted to studying advanced region merging heuristics, to use of Bezier curves and filter enhancement.

\section{References}

1. Duce, D., Herman, I., Hopgood, B.: Web 2D Graphics File Format. Computer Graphics forum 21(1) (2002) 43-64

2. Rabaud, V., Belongie, S.: Big Little Icons. In: CVAVI, San Diego CA (2005)

3. Vantighem, C., Laurent, N., Deckeur, D., Plantinet, V.: Vector eye 1.0.7.6 (2003) Copyright SIAME e Celinea, http://www.siame.com, http://www.celinea.com.

4. Weber, M.: Autotrace 0.31 (2002) GNU General Public License, http://www. autotrace.sourceforge.net.

5. Kuhl, K.: Kvec 2.99 (2003) Copyright KK-Software, http://www.kvec.de.

6. Battiato, S., Costanzo, A., Di Blasi, G., Nicotra, S.: SVG Rendering by Watershed Decomposition. In: Proceeding of SPIE Electronic Imaging-Internet Imaging VI-. Volume 5670.3. (2005)

7. Battiato, S., Barbera, G., Di Blasi, G., Gallo, G., Messina, G.: Advanced SVG Triangulation Polygonalization of Digital Images. In: Proceeding of SPIE Electronic Imaging-Internet Imaging VI-. Volume 5670.1. (2005)

8. Battiato, S., Di Blasi, G., Gallo, G., Messina, G., Nicotra, S.: SVG Rendering for Internet Imaging. In: Proceeding of IEEE CAMP'05, International Workshop on Computer Architecture for Machine Perception, Palermo(Italy) (2005) 333-338

9. Prasad, L., Skourikhine, A.: Raster to Vector Conversion of Images for Efficient SVG Representation. In: Proceedings of SVGOpen'05, NL (2005)

10. Nock, R., Nielsen, F.: Statistical Region Merging. IEEE Transaction on Pattern Analysis and Machine Intelligence 26(11) (2004) 1452-1458

11. Lucchese, L., Mitra, S.: Color Image Segmentation: A State-of-the-Art Survey. In: Proc. of the Indian National Science Academy(INSA-A). Volume 67 A. (2001) 207-221

12. World Wide Web Consortium: Scalable Vector Graphics (SVG) 1.1 Specification. (2003) http://www.w3.org/TR/2003/REC-SVG11-20030114/. 